\title{
Implementation and Results of New High Resolution SAR Modes for an Airborne Maritime Patrol Radar
}

\author{
Damian Gromek, Piotr Samczyński, Jacek Misiurewicz, Mateusz Malanowski, Krzysztof Kulpa, Artur Gromek, \\ Andrzej Gadoś, Anna Jarzębska, and Maciej Smolarczyk
}

\begin{abstract}
The paper presents new high resolution SAR results of real-life measurements using an updated ARS-400/ARS-800 SAR sensor installed on the maritime patrol aircraft M-28. The main role for such radars is surveying the sea surface, and the imaging of selected targets (e.g. ships, roads, vehicles, buildings, etc.) to help the operator in classifying them. In the present day increasing computing power, improved algorithms and general technological progress has allowed the obtaining of better results in SAR imagery.
\end{abstract}

Keywords-high resolution SAR, real-time SAR imaging, ISAR, surveillance radar

\section{INTRODUCTION}

$\mathbf{M}$ ARITIME patrol aircraft are used in Poland to survey large Baltic sea areas, detect, track and identify vessels in the Exclusive Economic Zone, and to identify any unexpected behavior of the targets. It is used in all kinds of missions such as exploration, rescue, patrol and reconnaissance. The patrolling of large sea and ground areas is the basic task of the Coast Guard, and also of the Navy in special missions. This can be done through the use of an SAR sensor [1], [2], [3] installed on the Polish plane M-28, which is used by both organizations. The on-board SAR system is the multimode X-band maritime radar with surveillance and SAR (Synthetic Aperture Radar) imaging modes. The SAR sensor was developed by Polish scientists from the Telecommunications Research Institute and the Warsaw University of Technology [4].

The government organizations mentioned above use the SAR sensor for sea surface monitoring to detect illegal immigration, illegal fishing, oil slicks, and activities against the environment. Over the ground, they are capable of monitoring rivers in the event of high water levels and floods, forests, areas of vegetation, grasslands, as well as urban and industrial infrastructure. SAR processing is implemented in real-time. The paper presents how modern technology - new algorithms, better hardware and the increase in computer power - has been used to update the sensor in order to obtain high resolution SAR images.

This work was supported by the Polish Ministry of Science and Higher Education under the Development Grant no. 0182/R/T00/2010/12.

D. Gromek, P. Samczyński, J. Misiurewicz, M. Malanowski, K. Kulpa A. Gromek are with the Institute of Electronic Systems, Warsaw University of Technology, Nowowiejska 15/19, Warsaw, Poland (e-mail: dgromek@stud.elka.pw.edu.pl).

A.Gadoś, A. Jarzebska, and M. Smolarczyk are with Bumar Elektronika S.A., Poligonowa 30, 04-051 Warsaw, Poland.
The Polish maritime surveillance plane M-28 (Fig. 1) is equipped with an $\mathrm{X}$-band multifunction radar with an instrumented range of $240 \mathrm{~km} \mathrm{[4],} \mathrm{[5],} \mathrm{[6],} \mathrm{[7].} \mathrm{In} \mathrm{the} \mathrm{surveillance}$ mode, the radar is able to detect small targets in high seas, and thus it is often used for Search and Rescue missions. On these missions, it is very important to be able to distinguish between different types of objects in order to find the one of greater importance to the mission. For this purpose, SAR modes are used to obtain high quality images of the target. Special SAR modes are also used for detecting sea surface pollution, the present state of the coast line (especially after heavy storms, where change detection plays an important role) and to monitor the ice cover in the coastal zone.

These days, the emphasis in air missions is on recognition capability rather than detection only. For the purpose of recognition and classification of sea targets, a combination of methods can be applied (multi-sensors detection for military application, and radar multi-mode operation for civilian application). The Polish maritime patrol aircraft M-28 is a multisensor system, and its radar sensor is also a multi-mode system with the following capabilities [4], [5]:

- surveillance mode with rotating antenna - instrumented ranges from 7 to $240 \mathrm{~km}$,

- stripmap SAR mode with stopped antenna $-15 \times 15 \mathrm{~m}$ resolution,

These modes allow both detection and recognition of targets, shapes, and areas, and they increase the effectiveness of air missions.

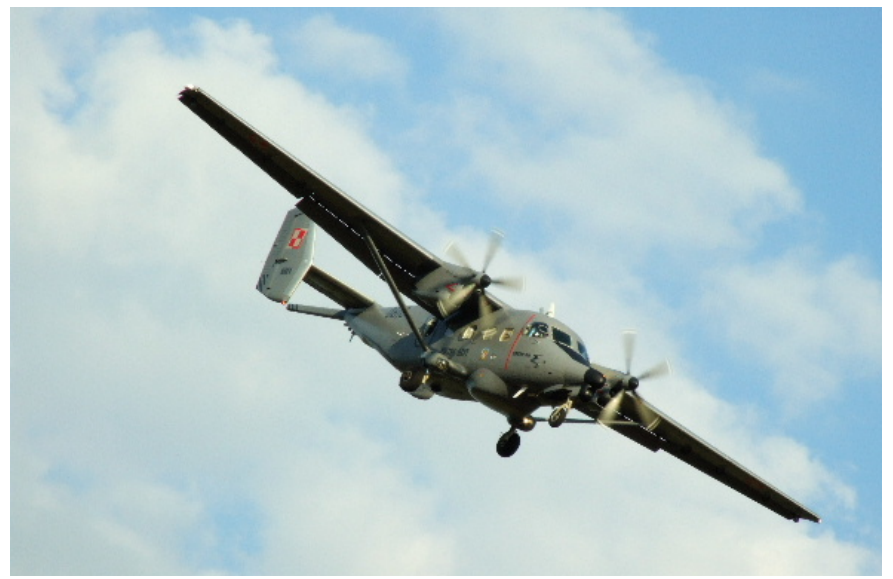

Fig. 1. The Polish Navy M28 aircraft with maritime patrol radar. 


\section{OPERATION MODES}

Planning of the mission is the most important aspect during surveillance operations. The mission is carried out not only on a designated route; it also takes into account the situation on the sea surface obtained on board the aircraft. Of most interest to a surveillance operation are targets which display behavior that is unusual. For example, targets that are maneuvering in prohibited areas, non-cooperating targets or those sending false identification data via Automatic Identification Systems (AIS). An airborne radar system operating in several different modes gives an opportunity to assess the situation and undertake a comprehensive diagnosis.

Each mode of airborne radar gives additional information of the detected objects. The surveillance mode allows the user to quickly detect objects over a very large area (maximum scan area is about 1.5 thousand $\mathrm{km} 2$ ). The analysis of the image obtained in the patrol mode gives the operator the opportunity to select regions of interest, and to identify them for scanning in the SAR mode. The SAR image of the area is performed at a low resolution to obtain an image with low detail, but covering a large area (image resolution is approximately $15 \times 15 \mathrm{~m}$ ). A low resolution SAR image allows the user to perform a preliminary classification of the object's type. During this step the user can separate fishing nets from ships, groups of surface targets, and make an initial classification of the objects. Additionally, the radar can receive information from the AIS system. This allows the separation of cooperating and noncooperating objects. By combining the information above, the user can decide which of them may be suspects.

The update of the radar described below adds the possibility of performing a second classification step using a high resolution SAR image. In the result of the update, high resolution of the sensor has been obtained both in the range and cross-range directions.

\section{High RANGE RESOLUtion}

The current SAR mode available on the ARS400M/800 radar has a resolution in both the range and cross-range directions of 15 meters. One of the goals of the project was to introduce a new high resolution SAR (HiSAR) mode with a resolution of up to 1.5 meters $x 1.5$ meters. The HiSAR mode operates in strip-map with the antenna perpendicular to the direction of the flight. This solution significantly increases the efficiency of terrestrial radar missions.

Improvement in range resolution is achieved by correlation reception and through the use of a chirp (LMF) sounding signal. In this case resolution depends only on the bandwidth B of the emitted chirp signal. In order to improve the range resolution of the ARS400M/800 radar the range dechirping technique (or deramping technique) was proposed and implemented (Fig. 2). The deramping technique allows the achievement of the practical range resolution of 0.5 to $2 \mathrm{~m}$ and above, which allows the user to have a rather good quality of images under both the SAR and ISAR modes. In this technique the cost of obtaining high range resolution is the narrow strip of scanned area by the radar (Fig. 3). It can be described as a simple ZOOM technique. The big advantage

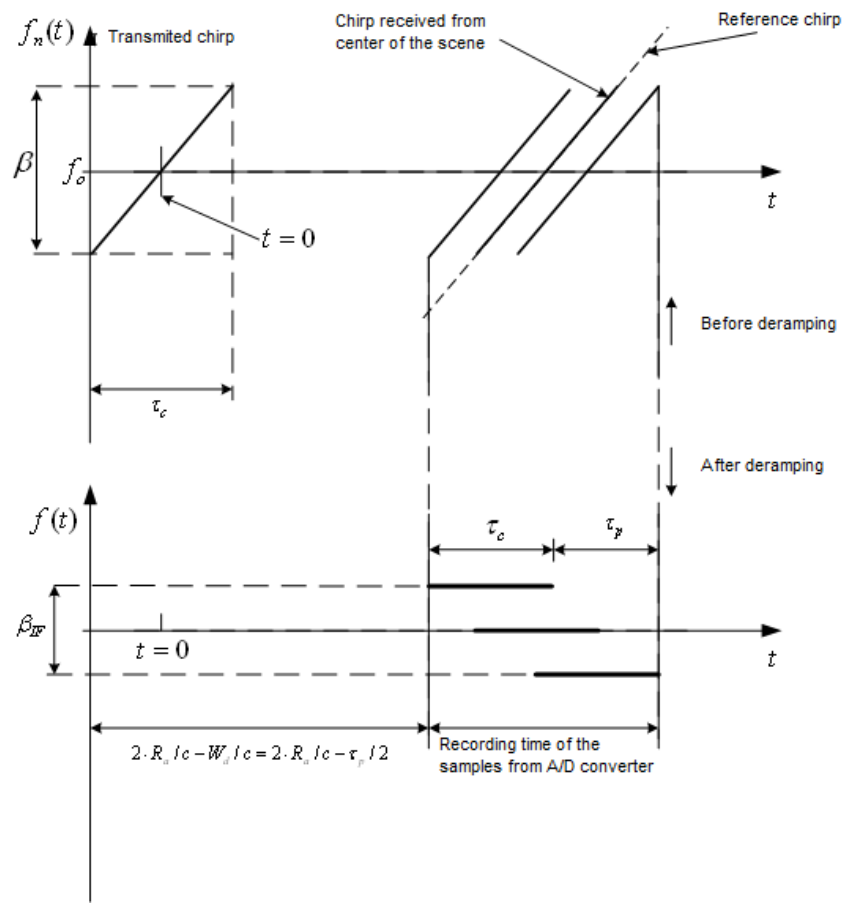

Fig. 2. Deramping technique.

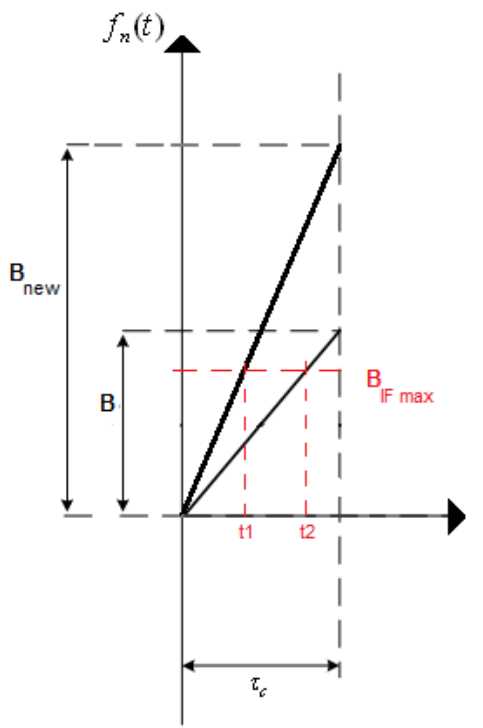

Fig. 3. Increased bandwidth - means narrow strip scaned - ZOOM.

of the deramping technique is the reduction of the bandwidth of the received signal from the narrow strip illuminated by the radar. Bandwidth reduction is achieved by mixing the received chirp with a replica of the transmitted chirp at a given time delay. The reference chirp is delayed in time by the value of the pulse propagation time between the antenna and the center of the analyzed scene. In the technique presented in Fig. 2, limited bandwidth of the receiver is no longer a problem for high resolution SAR images. By using this technique the received signal bandwidth is determined mainly by the width of the strip analyzed by the radar and by a chirp rate factor. 


\section{High AZIMUth Resolution}

High resolution in the azimuth direction can be obtained using advanced autofocus techniques for synthetic aperture radar (SAR autofocus) [8], [9], [10], [11]. Autofocusing algorithms use additional motion estimation and motion compensation of the radar platform movement (airplane) from the received radar data. The SAR radar technique theoretically allows for resolution in azimuth to be equal to half of the length of a real antenna's aperture. For the ARS400M/800 radar, the achievable theoretical azimuthal resolution is about $1.5 \mathrm{~m}$. However, achieving such resolution in azimuth is not a trivial task and requires complex signal processing including autofocus. For example, in order to obtain a fully focused SAR image at a distance of about $10 \mathrm{~km}$, the required radar along-track velocity accuracy is $0.08 \mathrm{~m} / \mathrm{s}$. Navigation systems installed on board of the Bryza-1RM/Bis is highly insufficient. This is a very strict requirement and without the use of any additional processing, virtually impossible to meet.

There are two classes of autofocus techniques: parametric and nonparametric. Nonparametric are called PG - phase gradient and PD - phase difference. Parametric are CO contrast optimization and MD - map drift. In the project map drift autofocus algorithm [11] was used. A simplified block diagram is presented in Fig. 4.

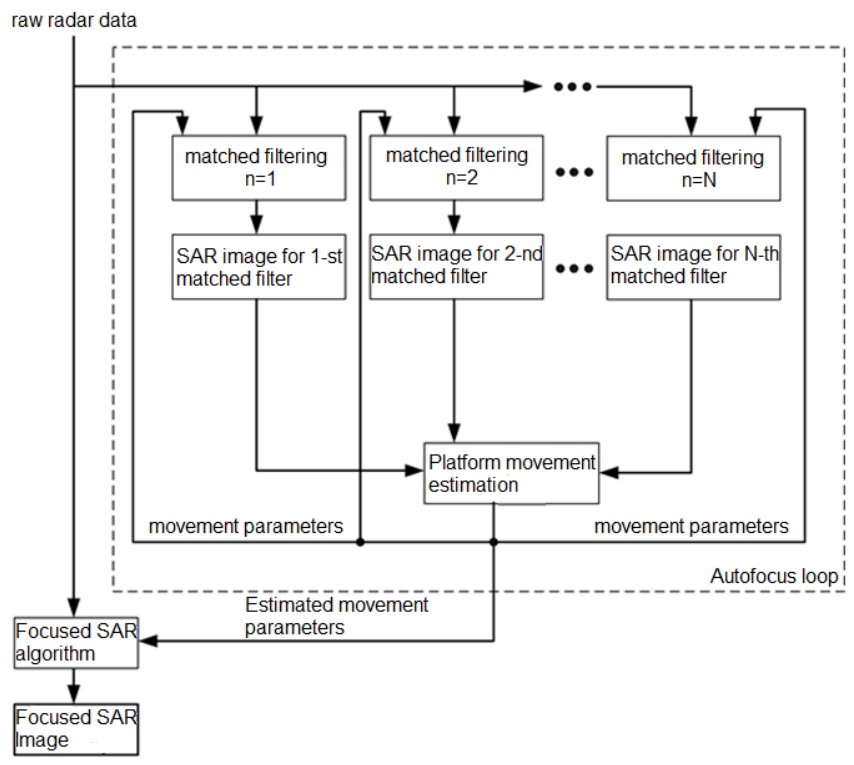

Fig. 4. Simplified block diagram of mapdrift algorithm.

MD algorithm has the least computational complexity and requires the smallest number of iterations to achieve a relatively good quality SAR image. MD divides the matched filter in azimuth into $\mathrm{N}$ matched subfilters. This technique is called multilook, after which an SAR image is produced from each subfilter. The comparison between SAR images produces a correction factor for each subfilter. In the next iteration MD uses corrected filters and creates new SAR images. Iterations are performed until the difference between SAR images is smaller than the set threshold. Usually 2-3 iterations are sufficient to obtain a reasonably good focused SAR image.

\section{EXAMPLE RESULTS}

Figure 5 shows a drilling platform in the Baltic Sea. In Fig. 6 the high resolution SAR image of that object is presented. For that purpose $100 \mathrm{MHz}$ signal bandwidth was used. At the obtained high resolution SAR image it can be easily distinguished between characteristic elements of the platform, such as the place for landing for helicopters, metal masts or cranes. In Figs. 7-10 are presented the azimuth and range

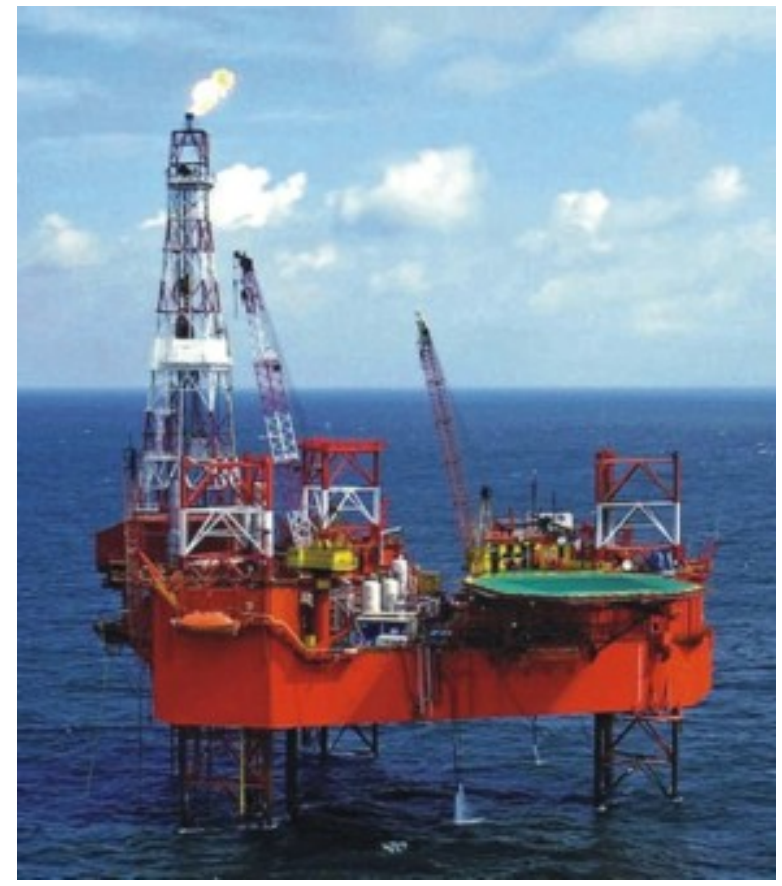

Fig. 5. Drilling platfrom on Baltic sea.

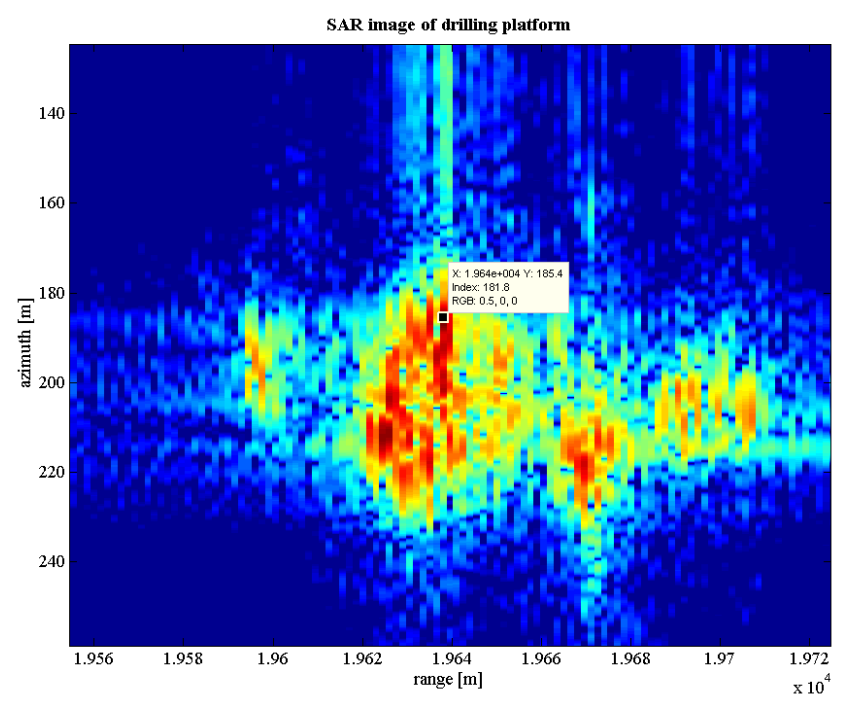

Fig. 6. Sar image of drilling platform.

direction profiles. Presented profiles are cross-sections through a point which is marked in Fig. 6. As shown in the zoomed peak value in azimuth profile presented in Fig. 8, the width of that peak is approximately $3.5 \mathrm{~m}$, which is only slightly lower than the assumption of $2 \mathrm{~m}$ azimuth resolution. Explanation 


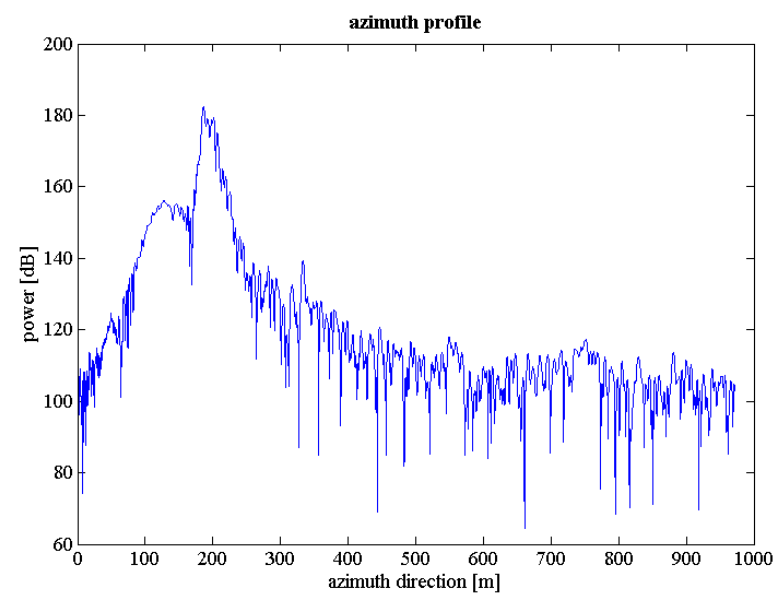

Fig. 7. Azimuth profile.

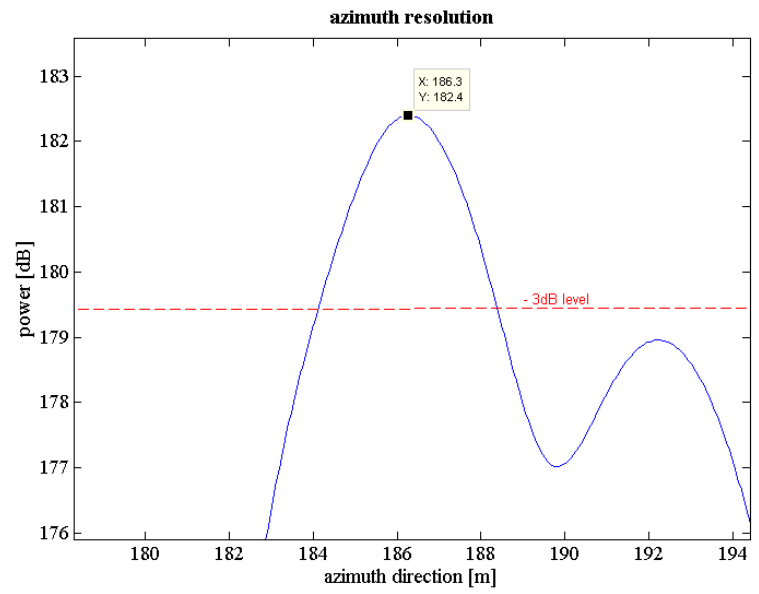

Fig. 8. Azimuth resolution - zoom.

might be in Fig. 7. The echo is an echo from a complex object - it is hard to find a single scatterer in real data - which may have an impact on azimuth resolution degradation. Figure 10 presents zoomed peak value in range direction profile. The visible peak has a width of about 1 meter. It follows that, even in the range direction the intended resolution was achieved. With a bandwidth of $100 \mathrm{MHz}$ theoretical resolution is $1.5 \mathrm{~m}$. It should be noted that the specificity of the object can have some influence on the results, so the results are better than expected. In this experiment, an object should be treated as complex, taking into account the fact that reflectivity of its components should be described by complex values.

In Fig. 11 the obtained SAR image of Gdynia's city seaport in the high resolution mode is presented. The chirp signal bandwidth was set to $100 \mathrm{MHz}$, so the resolution in the range direction was about 1.5 meters, and the same in the azimuth direction. For comparison, Fig. 12 shows the SAR image of the same area but at a much lower resolution. Bandwidth of the chirp signal was set to $12 \mathrm{MHz}$. The level of detail in the radar image acquired under the high resolution radar mode is much

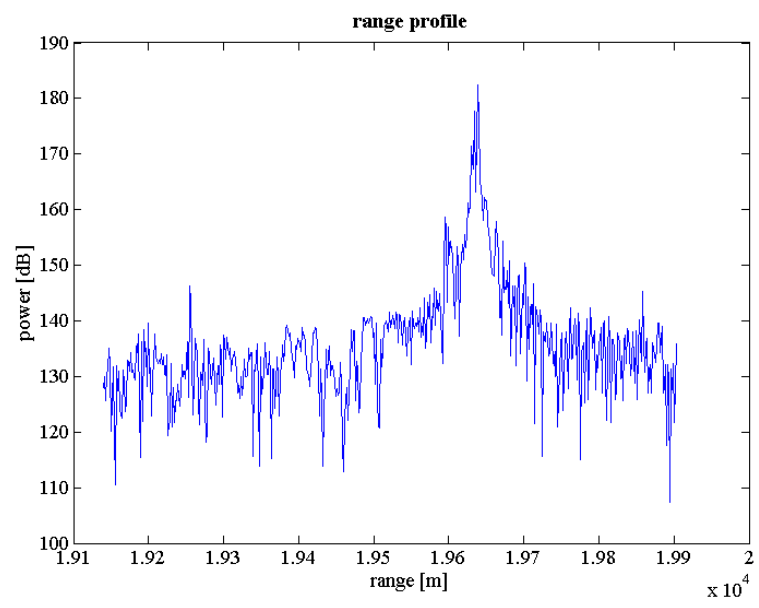

Fig. 9. Range profile.

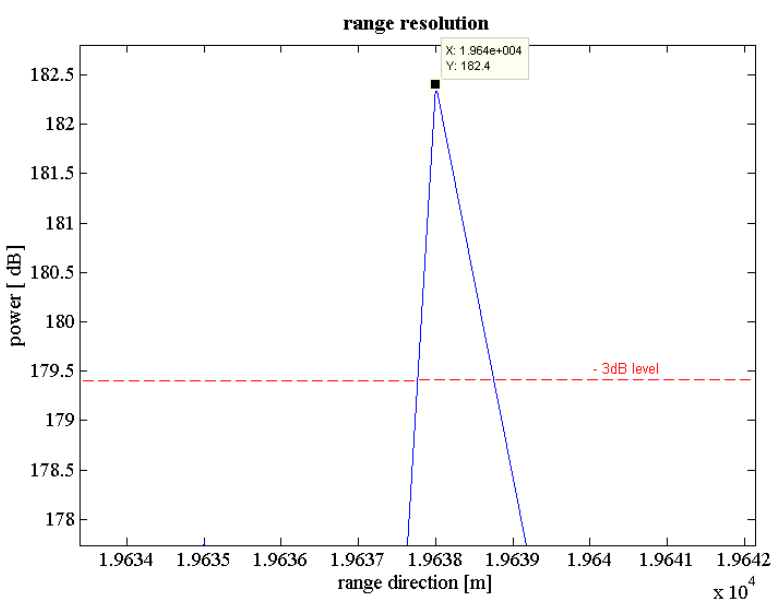

Fig. 10. Range resolution - zoom.

better than in the low resolution mode. Figure 13 presents an optical image of that area extracted from google maps. From comparison of images 11,12 and 13 it can be easily seen that target calssification is easier in high resolution mode. In high resolution SAR image it can be easily distinguish between building, ships, vehicles, roads and so on. This image contains much more information for further analysis.

\section{CONCLUSIONS}

To conclude, the presented modifications of the current maritime patrol radar system allows the achievement of very good results in the HiSAR operation mode. The use of deramping techniques and modern algorithms to compress the signal in the azimuth and range directions significantly improved the obtained resolution. It should also be noted that the radar operates in X-band, which is a range not typically used to obtain high resolution images. Despite this fact, the results are highly satisfactory. 


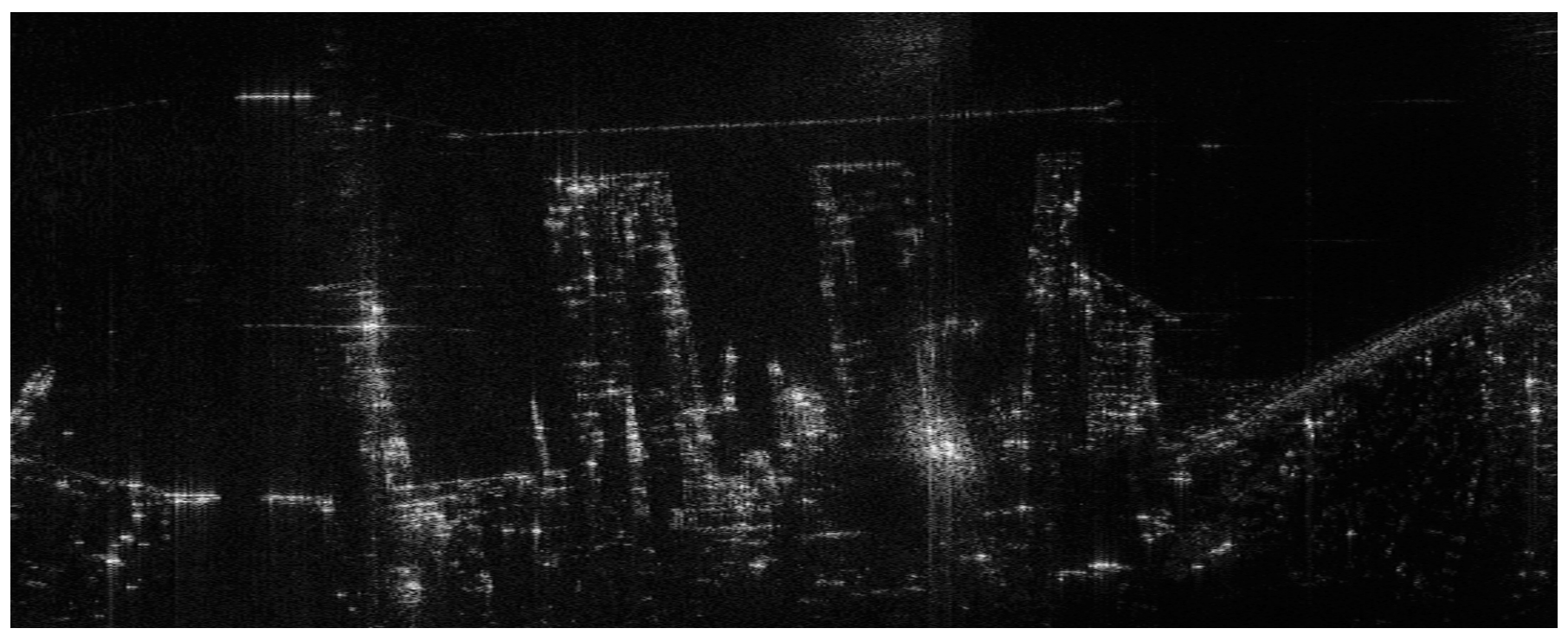

Fig. 11. High resolution SAR image of Gdynia's seaport

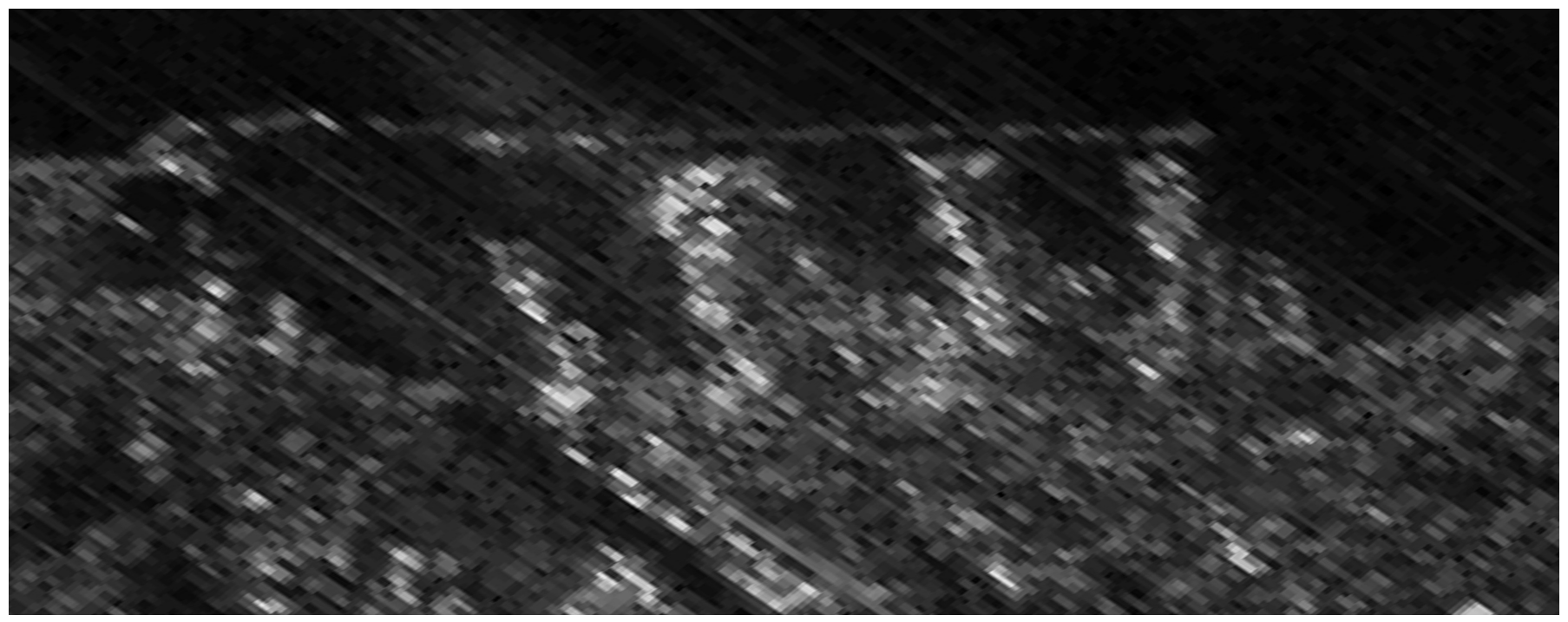

Fig. 12. Low resolution SAR image of Gdynia's seaport

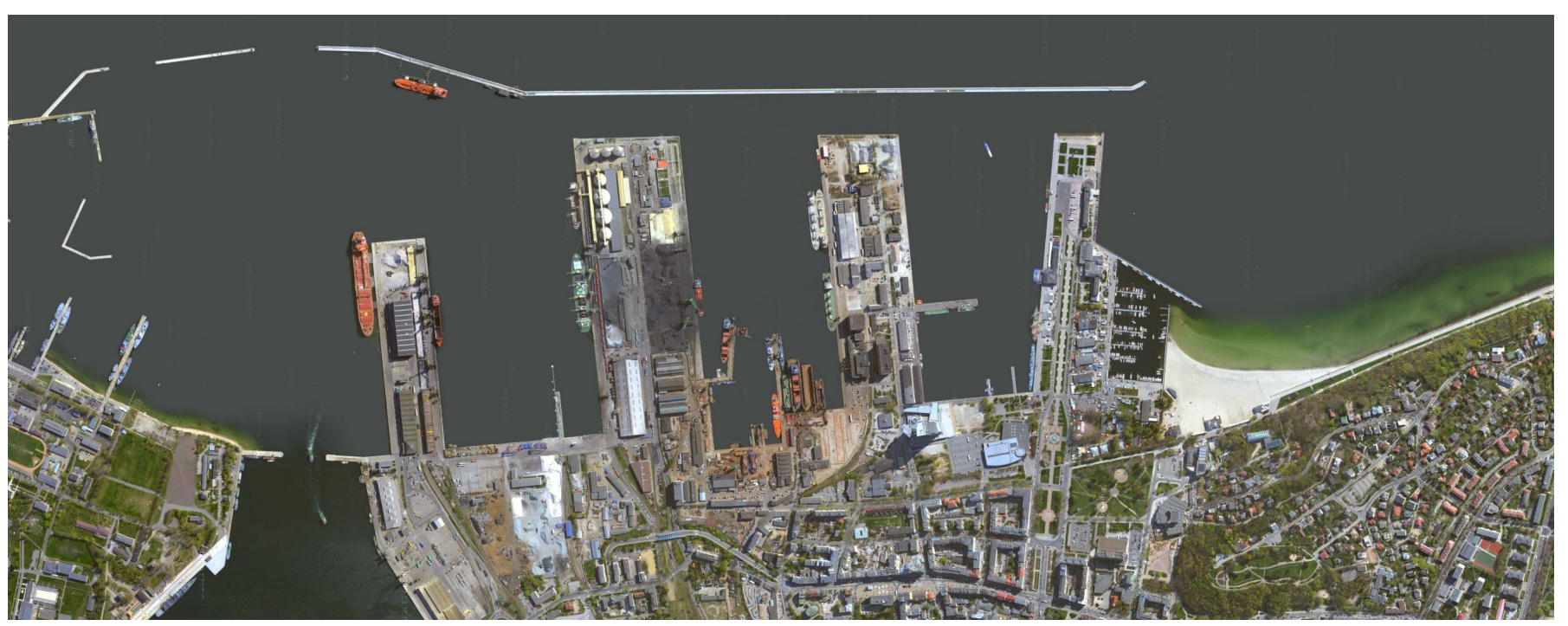

Fig. 13. Optical image of Gdynia's seaport (GoogleMaps) 


\section{REFERENCES}

[1] G. Granceschetti and G. Lanari, "Synthetic aperture radar processing," crc press llc, 1999.

[2] J. C. Curlander and R. N. Mcdonough, "Synthetic aperture radar systems and signal processing," john wiley and sons, 1991.

[3] C. Oliver and S. Quegan, "Understanding synthetic aperture radar images," Artech House, 1997.

[4] J. Piotrowski and K. Kulpa, "The sea pollution monitoring in the first polish multifunction maritime patrol system," Proc. Third Int. Airborne Remote Sensing Conf. Exhibition, vol. II, pp. 173-179, July 1997.

[5] A. Gadoś, A. Gorzelańczyk, A. Jarzebska, M. Mordzonek, M. Smolarczyk, K. S. Kulpa, and B. Dawidowicz, "First polish sar trials," Proc. EUSAR, vol. I, pp. 279-282, May 2004, ulm, Germany.

[6] P. Samczyński, A. Gadoś, K. Kulpa, M. Malanowski, J. Misiurewicz, M. Mordzonek, and M. Smolarczyk, "Signal processing of airborne maritime patrol radar," IQPC Military Sensors 2009 Conference, November 2009, london, UK
[7] A. Gadoś, A. Jarzebska, M. Smolarczyk, K. Kulpa, M. Malanowski, J. Misiurewicz, and P. J. Samczyński, "Bryza-1rm/bis - multimission polish navy plane with sar sensor dedicated to sea and ground monitoring," Proceeding of APSAR 2011, pp. 1-5, 2011.

[8] K. Kulpa, J. Misiurewicz, P. Samczyński, and M. Smolarczyk, "Multilook technique for dominant scatterer removal in sar images," 3rd World Congress 'Aviation in the XXI-st Century' - MRRS-2008 Symposium Proceedings, September 2008, kiev, Ukraine.

[9] P. Samczyński, K. S. Kulpa, J. Misiurewicz, and M. Smolarczyk, "Identification of building wall orientation angle with multilook sar processing," Journal of Acta Physica Polonica A, vol. 119, no. 4, 2010.

[10] P. J. Samczyński, "Super-convergent velocity estimator for an autofocus coherent mapdrift technique," IEEE Geoscience and Remote Sensing Letters, vol. PP, no. 99, pp. 1-5, 2011, iEEE Geoscience and Remote Sensing Society.

[11] —_, "Koherentna technika autofocus mapdrift," Krajowa Konferencja Radiokomunikacji Radiofonii i Telewizji, p. 66, 2011, katedra Radiokomunikacji, Politechnika Poznańska. 